Artigo Original

\title{
Tradução e validação da adaptação para o exercício do Perceived Motivational Climate Sport Questionnaire
}

\author{
Luís Cid ${ }^{1,4}$ \\ João Moutão ${ }^{1,4}$ \\ José Leitão 2,4 \\ José Alves ${ }^{3,4}$ \\ ${ }^{1}$ Escola Superior de Desporto de Rio Maior (ESDRM), Rio Maior, Portugal \\ ${ }^{2}$ Universidade de Trás-os-Montes e Alto Douro (UTAD), Vila Real, Portugal \\ ${ }^{3}$ Instituto Superior de Ciências da Saúde - Norte (ISCS-N), Granda, Portugal \\ ${ }^{4}$ Centro de Investigação em Desporto, Saúde e Desenvolvimento Humano (CIDESD), \\ Vila Real, Portugal
}

\begin{abstract}
Resumo: O objetivo principal do estudo é a tradução e validação da versão portuguesa da adaptação ao exercício do Perceived Motivational Climate in Sport Questionnaire (PMCSQ), com recurso à análise fatorial exploratória (AFE) e confirmatória (AFC), realizadas com dois grupos independentes de praticantes de exercício em ginásios, de ambos os géneros e com idades compreendidas entre os 14 e os 64 anos. Na AFE os resultados revelam uma estrutura que explica $52 \%$ da variância dos resultados, pesos fatoriais entre 0.63 e 0.80 , e uma boa consistência interna $\left(\alpha_{\text {Mestria }}=0.78\right.$; $\left.\alpha_{\text {Performance }}=0.74\right)$. Na AFC os resultados indicam um excelente ajustamento do modelo: $S-B X^{2}=40.6 ; d f=34 ; p=0.20 ; S-B X^{2} / d f=1.19 ; S R M R=0.03$; $\mathrm{NNFI}=0.98 ; \mathrm{CFI}=0.99 ; \mathrm{RMSEA}=0.02 ; 90 \%$ IC RMSEA=0.00-0.05, uma consistência interna razoável $\left(\alpha_{\text {Mestria }}=0.74 ; \alpha_{\text {Performance }}=0.75\right)$, e pesos fatoriais entre 0.50 e 0.79 , o que nos leva a concluir que a versão Portuguesa da adaptação do PMCSQ ao exercício pode ser utilizada na avaliação do clima motivacional no exercício.
\end{abstract}

Palavras-chave: Objetivos de realização. Clima motivacional. Exercício. Avaliação psicológica.

\section{Translation and validation of the exercise adaptation of the Perceived Motivational Climate Sport Questionnaire}

\begin{abstract}
The main purpose of this study is to present the results of translation and validation of the Portuguese version of Perceived Motivational Climate in Sport Questionnaire (PMCSQ) adaptation to exercise, through exploratory (EFA) and confirmatory (CFA) factor analysis, performed with two independent groups of participants, all exercisers in private fitness clubs, of both sexes, and aged between 14 and 64 years old. The EFA results reveal a structure explaining $52 \%$ of total variance, with factor loadings ranged from 0.63 to 0.80 , and reasonable reliability $\left(\alpha_{\text {Mastery }}=0.78 ; \alpha_{\text {Performance }}=0.74\right)$. The AFC results showed an excellent model fit to data: $\mathrm{S}-\mathrm{B} X^{2}=40.6 ; \mathrm{df}=34 ; \mathrm{p}=0.20 ; \mathrm{S}-\mathrm{B} \chi^{2} / \mathrm{df}=1.19 ; \mathrm{SRMR}=0.03 ; \mathrm{NNFI}=0.98 ; \mathrm{CFI}=0.99$; RMSEA $=0.02 ; 90 \%$ IC RMSEA $=0.00-0.05$, an acceptable reliability ( $\alpha_{\text {Mastery }}=0.74 ; \alpha_{\text {Performance }}=0.75$ ), and factor loadings ranged from 0.50 to 0.79 , which leads us to conclude that Portuguese version of PMCSQ adaptation to exercise can be used to measure motivational climate in exercise.
\end{abstract}

Keywords: Achievement motivation. Motivational climate. Exercise. Psychological assessment.

\section{Introdução}

De acordo com a teoria dos objetivos de realização (AGT: Achievement Goal Theory) (NICHOLLS, 1984; 1989), a principal preocupação das pessoas em contextos de realização é demonstrar capacidade ou competência, conseguirem obter sucesso e evitarem o fracasso. Por essa razão, a competência "desempenha um papel central nesta teoria" (NICHOLLS, 1984), ou, tal como foi designado por Roberts (2001): "é a força energética" deste modelo.
No quadro conceptual original, desenvolvido no âmbito da educação, mais concretamente no contexto da sala de aula (MAEHR; NICHOLLS, 1980; AMES, 1984; NICHOLLS, 1984; DWECK, 1986; AMES; ARCHER, 1988; NICHOLLS, 1989; AMES, 1992), pressupõe-se que o indivíduo é um organismo que opera de forma racional, direciona os seus objetivos intencionalmente, e que as suas metas de realização são orientadas pelas suas crenças que, consequentemente, influenciam a sua tomada de decisão e o comportamento em contextos de realização. Nesta teoria, os objetivos de realização refletem a forma como a 
demonstração de competência é concebida pelo indivíduo, sendo propostos dois grandes tipos de objetivos, que refletem diferentes formas de os sujeitos julgarem a sua própria competência: Tarefa ou Ego (NICHOLLS, 1984; 1989), ou, utilizando outra terminologia, Mestria ou Performance (AMES, 1984; 1992).

Segundo o seu autor (NICHOLLS, 1984; 1989) e outros que aplicaram posteriormente este modelo teórico ao contexto do desporto (ROBERTS, 1984; DUDA, 1987; 1992; DUDA; NICHOLLS, 1992; ROBERTS, 1992), da educação física (GOUDAS; BIDDLE, 1994; PAPAIOANNOU, 1994; TREASURE; ROBERTS, 1994; BIDDLE, CURY et al., 1995; TREASURE; ROBERTS, 1995) e mais recentemente do exercício (GEORGIADIS; BIDDLE et al., 2001; KILPATRICK; BARTHOLOMEW et al., 2003; PETHERICK; MARKLAND, 2008), quando os sujeitos (e.g., praticantes de exercício) estão orientados para a tarefa (e.g., aumentar o seu volume de carga no exercício de supino em função daquilo que consegue levantar) a sua perceção de competência é julgada por critérios autorreferenciada, ou seja, as exigências da tarefa, o esforço empregue, e a melhoria do seu nível técnico, originam sentimentos de sucesso. Por outro lado, quando os sujeitos estão orientados para o ego (e.g., levantar mais volume de carga no exercício de supino do que outro praticante) a sua principal preocupação é demonstrar competência superior aos outros, ou seja, os sujeitos sentem-se altamente competentes e bem-sucedidos apenas quando mostram que são melhor que os outros (i.e., a sua perceção de competência é julgada por critérios normativos). Neste caso, a experiência da melhoria pessoal (e.g., aumento do volume de carga) e o esforço empregado no exercício pode não resultar numa perceção de competência se o sujeito não fizer melhor que os outros, ou, se tiver de se esforçar mais para conseguir os mesmos resultados (i.e., normalmente a sensação de competência também surge se 0 individuo consegue fazer melhor que os outros e não for preciso muito esforço para consegui-lo).

Esta teoria tornou-se a mais importante abordagem à motivação no desporto (ROBERTS, 2001), porque a forma como os sujeitos orientam os seus objetivos de realização, afeta não só a quantidade, mas sobretudo a qualidade da sua motivação, pois os resultados em termos comportamentais, afetivos e cognitivos são diferenciados em função de uma orientação motivacional para a Tarefa ou para o Ego (NTOUMANIS; BIDDLE, 1999). De acordo com vários autores, em especial aqueles que mais estudaram (e estudam) esta questão no contexto da atividade física (e.g. BIDDLE, 2001; DUDA, 2001; ROBERTS, 2001; DUDA; BALAGUER, 2007), uma orientação para tarefa conduz os sujeitos a comportamento e resultados de realização positivos (normalmente designados de padrões adaptativos) que incluem: um maior compromisso com a atividade, mais empenho e esforço na realização, maior persistência na tarefa (mesmo quando as coisas não correm bem), escolha de tarefas desafiadoras, e uma procura contínua de melhoria das suas competências. Por outro lado, uma orientação para o ego conduz os sujeitos a comportamentos e resultados de realização menos positivos (normalmente designados de padrões mal adaptativos), tais como: menos empenho e menor esforço empregue na atividade, uma maior preocupação acerca do seu nível de competência (revelando maiores níveis de ansiedade), escolha de tarefas muito difíceis (para não experienciar sentimentos negativos no caso de não conseguir realizá-las) ou muito fáceis (para garantir que consegue atingir o sucesso) e um menor compromisso com a atividade que, eventualmente, poderá conduzir ao abandono (principalmente se os sentimentos de incompetência persistirem no tempo).

Todavia, segundo Nicholls (1989), está subjacente à teoria dos objetivos de realização, que a adoção de um envolvimento para a tarefa ou para ego, numa determinada atividade e num dado momento, não é só uma função da tendência disposicional (pessoal), mas também das características do contexto de realização (situacional). $\mathrm{Na}$ opinião de alguns autores (DUDA, 2001; DUDA; BALAGUER, 2007), isto quer dizer, que existem dois grandes construtos incorporados no modelo: 1) perceção dos objetivos situacionais (i.e., perceção do clima motivacional: que é a forma como o sujeito perceciona o ambiente social e psicológico do contexto que é induzido pelos outros significativos - instrutores, praticantes, família, amigos, etc.; 2) orientação dos objetivos disposicionais (i.e., orientação motivacional: que é a tendência individual para orientar os objetivos em função dos critérios de sucesso). Por outras palavras, "as perspetivas disposicionais sobre a realização, 
associadas às influências dos agentes sociais, determinam o significado pessoal da realização, $e$ o padrão cognitivo, afetivo e comportamental que o sujeito exibe num determinado momento" (NTOUMANIS; BIDDLE, 1999).

Uma das maiores contribuições para os avanços do conhecimento e da investigação em Psicologia do Desporto e do Exercício, mais concretamente aquela que é realizada com base na teoria dos objetivos de realização, consiste no desenvolvimento de instrumentos de medida válidos e fiáveis dos construtos teóricos que lhes estão subjacentes (DUDA; WHITEHEAD, 1998). $E$ de facto, no contexto do desporto e da educação física escolar, têm surgido ao longo dos anos diferentes escalas que avaliam a perceção do clima motivacional induzido pelos treinadores (SEIFRIZ; DUDA et al., 1992; WALLING; DUDA et al., 1993; NEWTON; DUDA et al., 2000), pelos professores de educação física (GOUDAS; BIDDLE, 1994; BIDDLE; CURY et al., 1995; PAPAIOANNOU, 1998), pelos pais (WHITE; DUDA et al., 1992) e pelos pares (NTOUMANIS; VAZOU, 2005).

No entanto, ao contrário do que sucede no campo do desporto e da educação física escolar, a aplicabilidade da teoria dos objetivos de realização ao domínio do exercício é ainda muito escassa, e só recentemente recebeu uma atenção especial na literatura, quer ao nível disposicional (KILPATRICK; BARTHOLOMEW et al., 2003; PETHERICK; MARKLAND, 2008), quer situacional (MURCIA; BLANCO et al., 2006; THOMAS; BARRON, 2006; MURCIA; ROMÁN et al., 2008). Uma das razões para que tal aconteça tem a ver com a inexistência de instrumentos de medida válidos, que permitam avaliar os construtos subjacentes à teoria, especificamente no contexto do exercício, o que tem contribuído para diminuir a sua dimensão mais aplicada ou a utilização menos correta de instrumentos validados para o contexto do desporto. Seja como for, se esta questão já foi resolvida para o caso da avaliação da orientação dos objetivos de realização, com o desenvolvimento da Goal Orientation in Exercise Scale (GOES: KILPATRICK; BARTHOLOMEW et al., 2003) e da Goal Orientation in Exercise Measure (GOEM: PETHERICK; MARKLAND, 2008), o mesmo não se pode dizer da avaliação da perceção do clima motivacional. Por isso, uma das estratégias que tem sido utilizada pelos investigadores é a adaptação de instrumentos do contexto do desporto, para o contexto do exercício (e.g., MURCIA; BLANCO et al., 2006).

Para ultrapassar esta dificuldade, Thomas e Barron (2006), desenvolveram um questionário composto por 20 itens, adaptado do Perceived Motivational Climate in Sport Questionnaire (PMCSQ: SEIFRIZ; DUDA et al., 1992; WALLING; DUDA et al., 1993). No entanto, os autores apenas reportaram os valores da fiabilidade interna do instrumento (Clima Mestria - 10 itens: $\alpha=0.86$; Clima Performance -10 itens: $\alpha=0.77$ ), não fazendo qualquer tipo de referência à validade de construto do modelo na adaptação realizada.

Desta forma, será o objetivo principal do presente estudo, apresentar os resultados da validação para a população Portuguesa, de uma versão reduzida da adaptação do PMCSQ (THOMAS; BARRON, 2006), desde a sua tradução para a língua Portuguesa até à determinação das suas qualidades psicométricas, com o recurso à análise fatorial exploratória e confirmatória do modelo de medida do clima motivacional no domínio do exercício.

\section{Métodos}

\section{Participantes}

Para a análise fatorial exploratória, participaram 122 sujeitos $(n=122)$, de ambos os géneros (76 femininos; 46 masculinos), com idades compreendidas entre os 14 e os 54 anos $(\mathrm{M}=29.1 ; \mathrm{SD}=8.1)$, todos praticantes de exercício físico em academias ou health clubs de diversas atividades (38 musculação; 48 aulas de grupo step, indoor cycling, localizada, aeróbica; 36 cardiofitness), com uma frequência de prática de 1 a 6 sessões por semana $(M=3.1 ; S D=1.0)$, e uma média de $4.4 \pm 2.3$ horas de prática por semana (entre 2 e 12 horas). Todos os sujeitos tinham uma experiência de prática que variava entre 1 e 121 meses $(M=15.1 ; S D=21.5)$.

Para a análise fatorial confirmatória, participaram 358 sujeitos ( $n=358$ ), de ambos os géneros (246 femininos; 112 masculinos), com idades compreendidas entre os 14 e os 54 anos $(\mathrm{M}=31.4 ; \mathrm{SD}=11.2)$, todos praticantes de exercício físico em academias ou health clubs de diversas atividades (93 musculação; 165 aulas de grupo; 100 cardiofitness), com uma frequência de prática de 1 a 6 sessões por semana ( $M=2.9$; $S D=1.1)$, e uma média de $4.1 \pm 2.3$ horas de prática por semana (entre 1 e 12 horas). Todos os sujeitos 
tinham uma experiência de prática que variava entre 1 e 121 meses $(M=17.3 ; S D=24.8)$.

\section{Instrumentos}

Perceived Motivational Climate in Exercise Questionnaire (PMCEQ: THOMAS; BARRON, 2006). Este instrumento, que é uma adaptação para o exercício do Perceived Motivational Climate in Sport Questionnaire (SEIFRIZ; DUDA et al., 1992; WALLING; DUDA et al., 1993), é constituído por 20 itens, aos quais se responde numa escala tipo Likert com 5 alternativas de resposta, que variam entre o "Discordo Totalmente" (1) e o "Concordo Totalmente" (5). Posteriormente os itens são agrupados em 2 fatores (Mestria: envolvimento para a tarefa; Performance: envolvimento para o ego), que representam as formas distintas de perceção dos sujeitos sobre o clima motivacional no contexto de exercício, de acordo com a teoria dos objetivos de realização (ao nível situacional) (AGT: NICHOLLS, 1984; 1989). No modelo original, a adaptação realizada apresentou os seguintes níveis de consistência internas: fator Mestria $(\alpha=0.86)$ e fator Performance ( $\alpha=0.77)$. Para além destes valores, Thomas e Barron (2006) não apresentaram qualquer informação adicional sobre a validação desta medida.

\section{Procedimentos de recolha dos dados}

Após a permissão para recolher a informação junto dos praticantes, estes foram abordados de forma aleatória pelos investigadores e assistentes de investigação junto da área de receção, antes da sessão de exercício e durante os dias da semana, sempre ao final do dia, uma vez que é neste horário que se concentram nas academias a maior parte dos praticantes. Após uma breve explicação sobre os objetivos do estudo, o instrumento de avaliação foi aplicado de forma individual, sempre em condições semelhantes. Todos os participantes que concordaram fazer parte do estudo fizeram-no de forma voluntária e o consentimento informado foi obtido. O tempo de aplicação do questionário demorou aproximadamente 10 minutos.

\section{Procedimentos de tradução do questionário e redução do número itens}

Para a tradução e adaptação do instrumento da língua original (Inglês) para a língua Portuguesa, foram adotados procedimentos metodológicos sugeridos por ( $\underline{\mathrm{CID}}$; ROSADO et al., 2012), similares aos que são propostos por
Vallerand (1989) e aconselhados por Banville, Desrosiers e Genet-Volet (2000), para a tradução e validação transcultural de instrumentos de avaliação psicológica. No entanto, ao contrário do que é proposto por Vallerand (1989), não foi utilizada a técnica de tradução/retroversão (translation/back translation technique) (ver: BRISLIN, 1970), mas sim uma abordagem por comité de especialistas (committee approach) (ver: BRISLIN, 1980). Este procedimento não só não é inédito, como é recomendado por diversos autores, uma vez que pode representar uma melhoria da qualidade na avaliação dos aspetos semânticos dos instrumentos (FONSECA; BRITO, 2005). Além disso, este método tem a vantagem de permitir que os membros do comité consigam detetar mais facilmente os possíveis erros inerentes ao processo de tradução, através da cooperação dentro da especialidade de cada um (GEISINGER, 2003).

Assim sendo, após o pedido de autorização para realizar a tradução da PMCEQ para Português a um dos seus autores (Jennifer Thomas), o processo foi desenvolvido em seis etapas, desde a versão inicial até à versão final:

1) Tradução Preliminar: esta primeira etapa foi efetuada pelos investigadores com o auxílio de 3 tradutores com formação superior em Inglês-Português, da qual resultou a $1^{\underline{a}}$ versão do questionário (20 itens);

2) Primeiro Painel de Avaliação: a análise/avaliação da versão inicial foi realizada individualmente por um júri composto por 4 especialistas de diferentes áreas do conhecimento científico (1 Licenciado em Línguas Português-Inglês, 1 Psicólogo, 2 Psicólogos do Desporto, 1 Licenciado em Ciências do Desporto). Das sugestões de alteração apresentadas, resultou a $2^{\underline{a}}$ versão do questionário (20 itens);

3) Segundo Painel de Avaliação: a $2^{a}$ versão do questionário foi outra vez submetida à análise/avaliação de outro júri (diferente das fases anteriores), desta vez composto por 4 especialistas (1 Psicólogo Clínico, 2 Psicólogos do Desporto, 1 Licenciado em Ciências do Desporto). Esta fase só terminou quando houve concordância entre os especialistas e a 
opinião de todos os membros do júri foi unânime em relação ao conteúdo dos itens da nova versão do questionário ( $3^{a}$ versão: 20 itens);

4) Terceiro Painel de Avaliação: após a primeira avaliação, submeteu-se a versão de 20 itens a um processo de avaliação do ajustamento dos itens aos respetivos fatores, com o objetivo de reduzir o número de itens do questionário, de modo a que fossem retidos apenas os itens mais relevantes e pertinentes, e que melhor refletissem os construtos latentes. Assim sendo, com base nas propostas metodológicas utilizadas por diversos autores (SEIFRIZ; DUDA et al., 1992; PAPAIOANNOU, 1994; PAPAIOANNOU, 1998; NEWTON; DUDA et al., 2000; NTOUMANIS; VAZOU, 2005), esta tarefa foi realizado com 0 recurso a 5 especialistas em Psicologia do Desporto com formação de base distintas (2 em Ciências do Desporto, 2 em Psicologia, 1 em ambas). Para tal, foi solicitado que associassem os itens aos respetivos fatores (clima para a mestria ou clima para a performance), e classificassem 0 seu grau de ajustamento ao mesmo, através de uma escala que variava entre 1 ("Muito Mau Ajustamento") e 5 ("Muito Bom Ajustamento"). Os critérios de retenção dos itens foram os seguintes: 1) avaliação unânime por parte dos especialistas; 2) nível de ajustamento 4 ("Bom Ajustamento") ou 5 ("Muito Bom Ajustamento") ao fator. No final desta etapa, da qual resultou a $4^{\underline{a}}$ versão do questionário, foram retidos 11 dos 20 itens, ou seja, foram eliminados 9 itens por levantarem dúvidas (3 quanto à dimensão; 6 quanto ao ajustamento) e não serem claros o suficiente no que se refere à sua ligação com variável latente que supostamente deveriam avaliar, o que consequentemente conduziu à discordância entre os especialistas e ao não cumprimento dos critérios de retenção estabelecidos;

5) Estudo Piloto: elaboração do primeiro layout do instrumento e aplicação da $4^{\underline{a}}$ versão do questionário a 50 sujeitos (estudantes do ensino superior na área da Psicologia do Desporto e do Exercício e de
Condição Física e Saúde no Desporto) para análise e determinação das dificuldades de compreensão e interpretação do conteúdo dos seus itens. Desta fase, resultou a $5^{\mathrm{a}}$ versão do questionário;

6) Revisão Final: revisão do Português (aspetos de sintaxe - ortografia, gramática e construção frásica), realizada por 2 Professores de Português e elaboração do layout final do questionário (versão final).

\section{Procedimentos para a análise fatorial exploratória (AFE)}

O método de extração utilizado foi o das componentes principais (principal component), seguido de uma rotação ortogonal (Varimax) dos fatores, para que se possa obter uma solução fatorial mais clara e objetiva, maximizando assim os pesos fatoriais dos itens (BROWN, 2006). Neste caso concreto utilizou-se uma rotação ortogonal porque, tendo em linha de conta o modelo teórico (AGT: NICHOLLS, 1984; 1989), pressupõem-se que os fatores não estejam correlacionados entre si, sendo então a mais indicada (KAHN, 2006; WORTHINGTON; WHITTAKER, 2006). Em suma, seguindo as sugestões de $\underline{\text { Cid, }}$ et al. (2012), que são recomendadas por diversos autores (PREACHER; MACCALLUM, 2003; BROWN, 2006; HAIR; BLACK et al., 2006; HENSON; ROBERTS, 2006; KAHN, 2006; WORTHINGTON; WHITTAKER, 2006; BLUNCH, 2008), utilizaramse os seguintes critérios de determinação dos fatores e retenção dos itens: a) Critério de Kaiser - fatores com valor próprio (eigenvalue) igual ou superior a 1 ( $E V \geq 1)$; b) Peso fatorial (fator loadings) dos itens igual ou superior a 0.5 $(F L \geq 0.50)$; c) Inexistência de itens com pesos fatoriais com alguma relevância ( $F L>0.30)$ em mais do que um fator (cross-loadings), mas se isso acontecer e a diferença entre eles for significativa (>0.15), o item deve ser eliminado; $d$ ) A percentagem da variância explicada pelos fatores retidos deve ser no mínimo de pelo menos 40\%; e) A consistência interna (alfa de Cronbach) do fator deve ser igual ou superior a 0.70 $(\alpha \geq 0.70) ; f) A$ consistência interna do fator não deve aumentar se um item for eliminado; g) Só devem ser retidos os fatores com pelo menos 3 
itens. A AFE foi realizada com recurso ao software de análise estatística SPSS 17.0.

\section{Procedimentos para a análise fatorial confirmatória ( $A F C$ )}

O método de estimação utilizado foi o da máxima verosimilhança (ML: Maximum Likeliood). No entanto, a teoria subjacente ao método de estimação ML assume que os dados tenham uma distribuição normal multivariada (KLINE, 2005; $\underline{K A H N}, 2006$ ), e quando isso não acontece (o que é o nosso caso), uma das soluções possíveis (BYRNE, 1994; $\underline{\text { HU; BENTLER, 1999; BYRNE}, ~}$ 2006; BENTLER, 2007) é a utilização do teste estatístico robusto do qui-quadrado, o chamado Satorra-Bentler $X^{2} \quad\left(S-B \quad X^{2}\right)$ (ver SATORRA; BENTLER, 1994), que corrige os valores para a não normalidade da distribuição dos dados, e produz resultados mais satisfatórios ( $\underline{\mathrm{CHOU}}$; BENTLER, 1995). Para além do teste S-B $X^{2}$, os respetivos graus de liberdade (df) e o nível de significância (p), foram ainda utilizados os chamados índices de ajustamento Standardized Root Mean Square Residual (SRMR), Comparative Fit Index (CFI), Non-Normed Fit Index (NNFI), Root Mean Square Error of Approximation (RMSEA) e o respetivo intervalo de

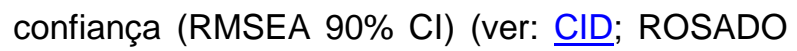
et al., 2012). No presente estudo, para os índices referidos, foram adotados os valores de corte sugeridos por $\underline{\mathrm{Hu}}$ e Bentler (1999): SRMR $\leq 0.08$, CFI e NNFI $\geq 0.95$ e RMSEA $\leq$ 0.06. A AFC foi realizada com o recurso ao software de análise de equações estruturais EQS 6.1 (BENTLER, 2002).

\section{Resultados}

\section{Análise Fatorial Exploratória (AFE)}

Em primeiro lugar, a medida de adequação da amostra (teste $\mathrm{KMO}=0.77$ ) indica que a análise de componentes principais pode ser realizada (valores acima de 0.60 indicam que a análise é boa), e o teste de esfericidade significativo (valores do teste de Bartlett associados a um $\mathrm{p}<0.05)$ diz-nos que as variáveis são correlacionáveis (HILL; HILL, 2000; PESTANA; GAGEIRO, 2005; WORTHINGTON; WHITTAKER, 2006; MAROCO, 2007).
Após a eliminação dos 9 itens na fase de validação de conteúdo (mencionada na metodologia), os restantes 11 itens foram submetidos a uma primeira AFE, da qual resultou uma estrutura fatorial de 2 fatores, com valores próprios de 2.81 (Mestria) e 2.51 (Performance), que explicam cerca de $48 \%$ da variância dos resultados, com pesos fatoriais que variam entre 0.32 e 0.76 , e uma fiabilidade interna de 0.73 (Mestria) e 0.70 (Performance). No entanto, um dos itens associados ao fator 1 (Mestria: “...todos querem dar o seu melhor."), não cumpriu todos os critérios estabelecidos, pelo que procedemos à sua eliminação do modelo.

Como se sabe, a decisão de eliminar ou não um item não é fácil, mas cabe sempre ao investigador (TABACHNICK; FIDELL, 1989). Por isso, é aconselhável que se leve em linha de conta o interesse em manter ou não as variáveis (KAHN, 2006), mas sobretudo, aquilo que nos diz - modelo teórico subjacente (HENSON; ROBERTS, 2006). Desta forma, a nossa decisão baseou-se em dois aspetos fundamentais: 1) em termos práticos (por questões de estimação do modelo) não é necessário ter 6 itens para avaliar uma variável latente, e as boas práticas ditam um número mínimo de 3 itens por fator (embora seja mais aconselhado 4) (HAIR; BLACK et al., 2006); 2) em termos teóricos, também não é sustentável que se mantenha um item que suscite ambiguidade de interpretação e, ao que tudo indica, o item não estava a ser interpretado pelos sujeitos com estando a avaliar exclusivamente a perceção do clima motivacional orientado para a mestria (tarefa).

Atendendo ao exposto, na tabela 1 são apresentados os resultados relativos à solução inicial da estrutura do questionário com 10 itens que foram encontrados com o recurso à $A F E$, após a eliminação do item acima mencionado. $\mathrm{Na}$ tabela são indicados apenas os pesos fatoriais relevantes e considerados como valor mínimo para poderem ser interpretados (0.30) (TABACHNICK; FIDELL, 1989; HAIR; BLACK et al., 2006; KAHN, 2006; WORTHINGTON; WHITTAKER, 2006). 
Tabela 1 Análise Fatorial Exploratória (com rotação ortogonal Varimax) da PMCEQp ( $n=122)$.

\begin{tabular}{|c|c|c|c|c|c|}
\hline Itens & Mín.-Máx. & $M \pm S D$ & Comunalidades & Fator 1 & Fator 2 \\
\hline Item 1 & $1-5$ & $2.96 \pm 0.96$ & 0.54 & & 0.71 \\
\hline Item 2 & $3-5$ & $4.13 \pm 0.67$ & 0.48 & 0.68 & \\
\hline Item 3 & $1-4$ & $2.57 \pm 0.94$ & 0.49 & & 0.69 \\
\hline Item 4 & $3-5$ & $4.19 \pm 0.63$ & 0.50 & 0.71 & \\
\hline Item 5 & $1-5$ & $2.68 \pm 1.12$ & 0.43 & & 0.66 \\
\hline Item 6 & $3-5$ & $4.06 \pm 0.65$ & 0.60 & 0.77 & \\
\hline Item 7 & $1-5$ & $2.23 \pm 0.99$ & 0.62 & & 0.80 \\
\hline Item 8 & $2-5$ & $4.02 \pm 0.61$ & 0.55 & 0.73 & \\
\hline Item 9 & $1-5$ & $2.25 \pm 1.00$ & 0.40 & & 0.63 \\
\hline Item 10 & $2-5$ & $4.09 \pm 0.76$ & 0.58 & 0.76 & \\
\hline & & \multicolumn{2}{|c|}{ Fator 1: Mestria } & \multicolumn{2}{|c|}{ Fator 2: Performance } \\
\hline \multicolumn{2}{|l|}{ № Itens } & \multicolumn{2}{|r|}{5} & \multicolumn{2}{|c|}{5} \\
\hline \multicolumn{2}{|c|}{ Valor próprio } & \multicolumn{2}{|r|}{2.77} & \multicolumn{2}{|c|}{2.43} \\
\hline \multicolumn{2}{|c|}{ \% Variância } & \multicolumn{2}{|r|}{$27.7 \%$} & \multicolumn{2}{|c|}{$24.3 \%$} \\
\hline \multicolumn{2}{|c|}{ a de Cronbach } & \multicolumn{2}{|r|}{0.78} & \multicolumn{2}{|c|}{0.74} \\
\hline
\end{tabular}

\section{Análise da Fiabilidade Temporal}

Com o objetivo principal de avaliar a fiabilidade temporal das respostas ao questionário por parte dos sujeitos, efetuamos uma análise teste-reteste (coeficiente de correlação $r$ de Pearson) baseada na aplicação do instrumento ao mesmo sujeito em dois momentos distintos no tempo (ver tabela 2). Embora não seja consensual, o tempo que medeia as aplicações pode variar entre 1 e 4 semanas (HILL; HILL, 2000; NIDEFFER; SAGAL, 2001; MOREIRA, 2004). No nosso caso utilizámos 4 semanas de intervalo entre as aplicações, e uma amostra de 40 sujeitos $(n=40)$, de ambos os géneros, todos alunos do ensino superior de cursos de Desporto, envolvidos na prática de atividade física não competitiva.

Tabela 2 Análise Correlacional Teste-Reteste $(n=40)$.

\begin{tabular}{lccc}
\hline Itens & M $\mathbf{S D}$ & Valor $\mathbf{r}$ & Valor $\mathbf{p}$ \\
\hline Item 1 (Pré - Pós) & $2.95 \pm 0.88-2.80 \pm 0.91$ & 0.79 & $0.000^{*}$ \\
Item 2 (Pré - Pós) & $4.03 \pm 0.66-3.90 \pm 0.55$ & 0.79 & $0.000^{*}$ \\
Item 3 (Pré - Pós) & $2.80 \pm 0.94-2.68 \pm 0.89$ & 0.78 & $0.000^{*}$ \\
Item 4 (Pré - Pós) & $4.28 \pm 0.55-4.25 \pm 0.59$ & 0.73 & $0.000^{*}$ \\
Item 5 (Pré - Pós) & $2.78 \pm 0.83-2.75 \pm 0.89$ & 0.88 & $0.000^{*}$ \\
Item 6 (Pré - Pós) & $3.85 \pm 0.48-3.83 \pm 0.47$ & 0.71 & $0.000^{*}$ \\
Item 7 (Pré - Pós) & $2.45 \pm 0.97-2.48 \pm 0.93$ & 0.82 & $0.000^{*}$ \\
Item 8 (Pré - Pós) & $4.05 \pm 0.50-3.98 \pm 0.53$ & 0.77 & $0.000^{*}$ \\
Item 9 (Pré - Pós) & $2.60 \pm 0.98-2.63 \pm 0.95$ & 0.85 & $0.000^{*}$ \\
Item 10 (Pré - Pós) & $4.05 \pm 0.60-4.00 \pm 0.51$ & 0.76 & $0.000^{*}$ \\
\hline Fator Performance (Pré - Pós) & $2.72 \pm 0.71-2.63 \pm 0.73$ & 0.92 & $0.000^{*}$ \\
Fator Mestria (Pré - Pós) & $4.05 \pm 0.37-3.99 \pm 0.33$ & 0.87 & $0.000^{*}$ \\
\hline
\end{tabular}

*p<0.001; Pré (Pré-Teste); Pós (Pós-Teste).

\section{Análise Fatorial Confirmatória (AFC)}

Como já foi mencionada anteriormente, a teoria subjacente ao método de estimação $\mathrm{ML}$ (maximum likelihood), assume que os dados tenham uma distribuição normal multivariada (KLINE, 2005; KAHN, 2006). De acordo com Byrne (2006), se o coeficiente Mardia (MARDIA, 1970) normalizado for superior a 5.0 é indicativo que os dados não têm uma distribuição normal multivariada, o que acontece no caso do presente estudo (kurtosis multivariada: Mardia $=14.3$, Mardia normalizado=8.8). Como tal, foram equacionadas medidas corretivas, utilizando-se 0 qui-quadrado corrigido (Satorra-Bentler $X^{2}$ : S-B $x^{2}$ ).

Tomando em consideração os valores de corte adotados (HU; BENTLER, 1999), os resultados obtidos permitem afirmar que 0 modelo da 
PMCEQp (2 fatores - 10 itens) se ajusta de forma excelente aos dados, confirmando-se assim a estrutura fatorial inicial preconizada com recurso à AFE: $S-B X^{2}=40.6 ; d f=34 ; p=0.202 ; S-B X^{2} / d f=1.19$; $\mathrm{SRMR}=0.03 ; \quad \mathrm{NNFI}=0.98 ; \quad \mathrm{CFI}=0.99$; RMSEA $=0.02 ; 90 \%$ IC RMSEA $=0.00-0.05$.

Os resultados indicam ainda uma correlação negativa não significativa $(r=-0.13)$ entre os dois fatores, assim como, uma consistência interna razoável em ambos os fatores ( $\alpha_{\text {Mestria }}=0.74$; $\alpha_{\text {Performance }}=0.75$ ). Além disso, como podemos observar na tabela 3 , os pesos fatoriais estandardizados dos itens (todos estatisticamente significativos para um $p<0.001$ ), variam entre 0.50 e 0.71 (fator Mestria) e entre 0.53 e 0.79 (fator Performance).

Tabela 3. Parâmetros Individuais Estandardizados dos Itens da PMCEQp ( $n=358)$.

\begin{tabular}{cccccc}
\hline Item (Fator) & Mín-Máx & M \pm SD & FL & EV & SMC \\
\hline Item 1 (P) & $1-5$ & $2.85 \pm 1.00$ & 0.60 & 0.80 & 0.36 \\
Item 2 (M) & $3-5$ & $4.16 \pm 0.61$ & 0.61 & 0.79 & 0.38 \\
Item 3 (P) & $1-4$ & $2.50 \pm 1.00$ & 0.63 & 0.78 & 0.39 \\
Item 4 (M) & $3-5$ & $4.24 \pm 0.56$ & 0.58 & 0.81 & 0.34 \\
Item 5 (P) & $1-5$ & $2.63 \pm 1.11$ & 0.55 & 0.83 & 0.31 \\
Item 6 (M) & $3-5$ & $4.11 \pm 0.63$ & 0.71 & 0.71 & 0.50 \\
Item 7 (P) & $1-5$ & $2.15 \pm 0.98$ & 0.79 & 0.61 & 0.63 \\
Item 8 (M) & $2-5$ & $4.02 \pm 0.60$ & 0.50 & 0.87 & 0.25 \\
Item 9 (P) & $1-5$ & $2.15 \pm 1.00$ & 0.53 & 0.85 & 0.28 \\
Item 10 (M) & $2-5$ & $4.11 \pm 0.66$ & 0.63 & 0.78 & 0.39 \\
\hline
\end{tabular}

P (Performance); M (Mestria); Mín-Máx (valor mínimo e máximo); M (média); SD (desvio padrão); FL (fator loading peso fatorial); EV (error variance - variância do erro de medida); SMC (squared multiple correlation - variância do item explicada pelo fator).

\section{Discussão}

\section{Análise Fatorial Exploratória (AFE)}

A solução preconizada pela AFE, apesar de ser uma versão reduzida (10-itens), apresenta uma estrutura concordante com o modelo original (PMECQ: THOMAS; BARRON, 2006), com dois fatores com valores próprios acima de um, que justificam no seu conjunto $52 \%$ da variância total dos resultados. Embora não exista um valor absoluto que se possa adotar para todas as aplicações, normalmente um valor na casa dos $60 \%$ é considerado muito satisfatório na área das Ciências Sociais (HAIR; BLACK et al., 2006). No entanto, a percentagem mínima da variância total dos itens explicada pelos fatores deve ser de pelo menos $40 \%$ (BLUNCH, 2008), o que se verifica no nosso estudo.

Relativamente aos resultados das comunalidades, de acordo com Henson e Roberts (2006) e Worthington e Whittaker (2006), o seu valor deve funcionar, sobretudo, como um guia de orientação útil para a tomada de decisão no que respeita à retenção dos itens, e não como critério principal da sua eliminação, sendo aconselhado valores acima de 0.50 (o que indica que uma boa parte da variância dos resultados de cada item é explicada pela solução fatorial encontrada) (HAIR; BLACK et al., 2006). No entanto, a hipótese de eliminação dos itens só deve ser fortemente equacionada com valores abaixo de 0.40 (WORTHINGTON; WHITTAKER, 2006), o que não se verifica no presente estudo.

No que respeita aos pesos fatoriais dos itens nos respetivos fatores, todos apresentam valores acima do critério estabelecido (0.50), ou seja, entre 0.68 e 0.77 (fator Mestria), e entre 0.63 e 0.80 (fator Performance). Como se pode observar, a maioria dos itens tem um peso fatorial acima de 0.70 (e os restantes estão muito próximos), o que indica uma estrutura muito bem definida (TABACHNICK; FIDELL, 1989; HAIR; BLACK et al., 2006). Por outro lado, também verificamos que o alfa de Cronbach apresenta valores de consistência internos considerados razoáveis (HILL; HILL, 2000; PESTANA; GAGEIRO, 2005; HAIR; BLACK et al., 2006) para ambos os fatores ( $\alpha_{\text {Mestria }}=0.78 ; \alpha_{\text {Tarefa }}=0.74$ ).

\section{Análise da Fiabilidade Temporal}

Como podemos observar na tabela 2 , as correlações das respostas dadas aos itens entre a primeira e a segunda aplicação variam entre 0.71 (item 6) e 0.88 (item 5). Os valores referidos na literatura como mínimo aceitável são de 0.70 (NIDEFFER; SAGAL, 2001; ALLWORTH; PASSMORE, 2008) ou até mesmo de 0.60 (VALLERAND, 1989). Assim, tomando em 
consideração a técnica estatística utilizada, em termos práticos (HILL; HILL, 2000; PESTANA; GAGEIRO, 2005; MAROCO, 2007) podemos considerar que as correlações encontradas são altas (valores entre 0.70 e 0.89 ) e muito altas (valores acima de 0.89 - o que acontece no caso do fator Performance), o que nos leva a concluir que a PMCEQp possui um elevado grau de fiabilidade temporal.

\section{Análise Fatorial Confirmatória (AFC)}

Os resultados obtidos através da AFC revelaram um excelente ajustamento do modelo aos dados, tendo as estimativas dos parâmetros individuais cumprindo todos os critérios previamente definidos. Apesar disso a variância total extraída (média da percentagem de variância extraída do conjunto dos itens de um fator) em cada um dos fatores foi demasiado baixa: $37 \%$ (fator Mestria) e 39\% (fator Performance). Ora isto significa que existe ainda uma quantidade considerável de variância dos itens que não é explicada pelo fator, ou seja, que eventualmente pode existir pouca convergência entre o conjunto dos itens em representar a variável latente (embora tenhamos encontrado valores aceitáveis de consistência interna em ambos os fatores). Seja como for, e apesar não existir uma regra rígida para os valores de corte sobre este indicador, é aconselhável que a variância total

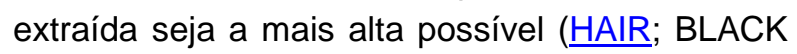
et al., 2006).

No entanto, ao analisarmos os resultados das validações do PMCSQ (AFE: DUDA; NICHOLLS, 1992; AFC: WALLING; DUDA et al., 1993), verificamos que existem as mesmas fragilidades nos instrumentos relativamente ao ajustamento do modelo (21-itens), senão vejamos: 1) na AFE foram extraídos 2 fatores (Mestria: 9 itens; Performance: 12 itens), que explicam no seu conjunto $39,7 \%$ da variância dos resultados, com pesos fatoriais entre 0.42 e 0.73 e valores de consistência interna de 0.84 (Performance) e 0.80 (Mestria); 2) na AFC, o modelo inicialmente preconizado na AFE não obteve um bom ajustamento $\quad\left(X^{2}=550.8 ; \quad \mathrm{df}=188 ; \quad \mathrm{p}=0.00\right.$; $X^{2} / \mathrm{df}=2.93 ; \mathrm{GFI}=0.77 ; \mathrm{AGFI}=0.72 ; \mathrm{RMSR}=0.11$ ), pelo que os autores consideraram que havia espaço para o seu melhoramento, após terem analisado os índices de modificação. No entanto, a reespecificação do modelo que resultou da correlação entre diversos erros de medida dos itens de cada fator (sem especificar quais), apresentou ainda algumas fragilidades $\left(X^{2}=346.1\right.$; $\mathrm{df}=171 ; \mathrm{p}=0.00 ; X^{2} / \mathrm{df}=2.02 ; \mathrm{GFI}=0.85 ; \mathrm{AGFI}=0.80 ;$ $\mathrm{RMSR}=0.09$ ). Além disso, apesar dos níveis de consistência interna dos fatores serem bastante aceitáveis $\quad\left(\alpha_{\text {Mestria }}=0.82 ; \quad \alpha_{\text {Performance }}=0.80\right), \quad$ o modelo continuou a apresentar uma quantidade elevada de variância não explicada, pelo que os seus autores consideraram que o modelo poderia ainda ser melhorado.

De acordo com Duda (2001), "a avaliação da perceção do clima motivacional no domínio da atividade física tem algumas limitações". Talvez seja devido à diversidade de subdimensões subjacentes ao quadro conceptual principal (sem excluir também as características psicossociais especificas dos contextos) que a investigação existente no campo da avaliação da perceção do clima motivacional revele alguma "derrapagem no conceito" (DUDA; WHITEHEAD, 1998). Em nossa opinião, esta questão pode ser comprovada pela análise dos resultados da validação de alguns dos instrumentos de medida desenvolvidos para avaliar a perceção do clima motivacional no desporto (PMCSQ-2: NEWTON; DUDA et al., 2000; PMCYSQ: NTOUMANIS; VAZOU, 2005), e na educação física (LAPOPECQ: PAPAIOANNOU, 1994; PECCS: BIDDLE; CURY et al., 1995), uma vez que ao serem constituídos por subescalas (fatores de $1^{\text {a }}$ ordem) subjacentes às duas escalas principais (fatores de $2^{\underline{a}}$ ordem: Ego e Tarefa), apresentam alguns problemas de ajustamento dos modelos. O que não se verifica nos questionários constituídos por uma estrutura fatorial com apenas as duas dimensões principais (CMI: MURCIA; BLANCO et al., 2006; MCSYS: SMITH; CUMMING et al., 2008).

De acordo com Duda e Whitehead (1998), apesar de tudo indicar que a perceção do clima motivacional possa ser multidimensional, os investigadores devem deixar claro quando as suas intenções são: a) explorar todas as variáveis que potencialmente influenciam a estrutura dos objetivos situacionais predominantes e ao mesmo tempo explicam a máxima proporção da variância dos resultados; ou b) identificar qual é o conjunto de variáveis que melhor se relacionam com as dimensões enfatizadas pelo modelo teórico, de modo a poder examinar quais os antecedentes e/ou consequências dos sujeitos percecionarem um clima motivacional para a Mestria ou para a Performance. Por isso, a nossa intenção foi validar um questionário que permitisse avaliar de forma clara e objetiva, as duas dimensões 
preconizadas pela teoria dos objetivos de realização no contexto do exercício. Não temos dúvidas de que a melhor opção foi a escolha de um instrumento "mais arrumado conceptualmente" e que restringisse a avaliação da perceção do clima motivacional a estas duas dimensões (DUDA; WHITEHEAD, 1998).

Em suma, como diz Biddle (2001), "do ponto de vista da avaliação, parece mais satisfatório a utilização de apenas dois fatores: Mestria e Performance". Por isso, se o objetivo do investigador é estudar o impacto que a perceção do clima motivacional tem sobre outras variáveis, então a melhor opção é a utilização do PMCSQ (no nosso caso a adaptação para o exercício) (ROBERTS, 2001). Segundo este autor é uma medida válida e fiável, que simplesmente avalia a perceção do sujeito face aos critérios de sucesso ou fracasso que estão inerentes ao contexto da realização, e como tal, "nunca devemos esquecer que a parcimónia e a elegância são atributos importantes na ciência" (ROBERTS, 2001).

Tomando em consideração o objetivo principal deste estudo, podemos concluir que o modelo de 2 fatores com 10 itens da versão Portuguesa da Perceived Motivational Climate in Exercise Questionnaire (PMCEQp) possui um bom ajustamento aos dados e, embora sendo uma versão reduzida, é consistente com o modelo original (PMCEQ: THOMAS; BARRON, 2006). Os resultados revelaram que a escala tem boas qualidades psicométricas que incluem os seguintes tipos de validade (BUCKWORTH; DISHMAN, 2002; HAIR; BLACK et al., 2006): a) Construto (exame ao "conceito" teórico que está subjacente ao teste), uma vez que cada item apresenta pesos fatoriais relevantes no fator onde deveria estar associado de acordo com o modelo teórico subjacente e o modelo de medida da versão original; b) Convergente (extensão pela qual os itens de um fator convergem ou partilham uma proporção elevada da sua variância comum), uma vez que os pesos fatoriais dos itens em cada um dos fator são elevados e significativos, bem como, a fiabilidade interna aceitável indica que todos os itens representam consistentemente a mesma variável latente); c) Discriminante (extensão pela qual os fatores são verdadeiramente distintos uns dos outros), uma vez que ao analisar os valores do teste de Langrage não se verificou a necessidade de adicionar parâmetros ao modelo (cross-loadings) para melhorar o seu ajustamento. Para além do ajustamento por via do teste de avaliação global (teste $\mathrm{S}-\mathrm{B} \mathrm{X}^{2}$ ), todos os outros índices de ajustamento (SRMR, NNFI, CFI, RMSEA) também atingiram os apertados valores de corte que são recomendados para evitar modelos desajustados (HU; BENTLER, 1999).

Em suma, as evidências encontradas garantem a validade e a fiabilidade da medida, pelo que a utilização deste questionário pode ser realizada sem qualquer reserva na avaliação do clima motivacional no contexto do exercício. Para além disso, pensamos que seria interessante a sua adaptação a outras culturas, pelo que encorajamos também a sua validação em outros países de língua portuguesa (e.g., o Brasil).

\section{Referências}

\section{ALLWORTH, E.; PASSMORE, J. Using psychometrics and psychological tools in coaching. In: PASSMORE, J. (Ed.). \\ Psychometrics in Coaching. Using Psychological and Psychometric Tools for Development. London: Kogan Page, p.7-25, 2008.}

AMES, C. Competitive, cooperative, and individualistic goal structure: A motivational analysis. In: AMES, R. e AMES, C. (Ed.). Research on motivation in education: Student motivation. New York: Academic Press, v.1, p.177-207, 1984.

AMES, C. Classrooms: goals, structure, and student motivation. Journal of Educational Psychology, Washington, v. 84, p. 261-271, 1992.

AMES, C.; ARCHER, J. Achievement goals in the classroom: Students learning strategies and motivation processes. Journal of Educational Psychology, Washington, v. 80, p. 260-267,1988.

BANVILLE, D.; DESROSIERS, P.; GENETVOLET, Y. Translating Questionnaires and Inventories Using a Cross-Cultural Translation Technique. Journal of Teaching in Physical Education, Champaign, v. 19, p. 374-387, 2000.

BENTLER, P. EQS 6 Structural Equations Program Manual. Encino, CA: Multivariate Software, Inc, 2002.

BENTLER, P. On tests and indices for evaluating structural models. Personality and Individual Differences, Oxford, v. 42, p. 825-829, 2007.

\section{BIDDLE, S. Enhancing Motivation in Physical}

Education. In: ROBERTS, G. (Ed.). Advances in Motivation in Sport and Exercise. Champaign: Human Kinetics, p.101-127, 2001. 
BIDDLE, S. et al. Development of scales to measure perceived physical education class climate: A cross-national project. British Journal of Educational Psychology, London, v. 65, p. 341-358, 1995.

\section{BLUNCH, N. Introduction to Structural Equation Modelling using SPSS and AMOS. London: Sage Publications, 2008.}

BRISLIN, R. Back translation for cross-cultural research. Journal of Cross-Cultural

Psychology, Thousand Oaks, v. 1, p. 185-216, 1970.

BRISLIN, R. Translation and content analysis for oral and written material. In: TRIANDIS, H. e BERRY, J. (Ed.). Handbook of Cross-Cultural Psychology. Needham Heights: Allyn and Bacon, v.2, p.389-444, 1980.

BROWN, T. Confirmatory Factor Analysis for Applied Research. New York: The Guiford Press, 2006.

\section{BUCKWORTH, J.; DISHMAN, R. Exercise}

Psychology. Champaign: Human Kinetics, 2002.

BYRNE, B. Structural Equation Modeling with EQS and EQS/Windows. Basic Concepts, Applications, and Programming. Thousand Oaks: Sage Publications, 1994.

BYRNE, B. Structural Equation Modeling with

EQS. Basic Concepts, Applications, and Programming. 2nd. New Jersey: Lawrence Erlbaum Associates, Publishers, 2006.

CHOU, C.; BENTLER, P. Estimates and tests in structural equation modeling. In: HOYLE, R. (Ed.). Structural Equation Modeling. Concepts, Issues, and Applications. Thousand Oaks, Thousand Oaks: Sage Publications, Inc., p.37-54, 1995.

CID, L.; ROSADO, A.; ALVES, J.; LEITÃO, J. Tradução e Validação de Questionários em Psicologia do Desporto e do Exercício. In: ROSADO, A., MESQUITA, I. e COLAÇO, C. (Ed.). Métodos e Técnicas de Investigação Qualitativa. Lisboa: Edições FMH, p. 29-64, 2012.

DUDA, J. Toward a Developmental Theory of Children's Motivation in Sport. Journal of Sport \& Exercise Psychology, Champaign, v. 9, n. 2, p. 130-145, 1987.

DUDA, J. Motivation in Sport Settings: A Goal Perspective Approach. In: ROBERTS, G. (Ed.). Motivation in Sport and Exercise. Champaign: Human Kinetics, p.57-91, 1992.
DUDA, J. Achievement goal research in sport: Pushing the boundaries and clarifying some misunderstandings. In: ROBERTS, G. (Ed.). Advances in Motivation in Sport and Exercise. Champaign: Human Kinetics, p.129-182, 2001.

DUDA, J.; BALAGUER, I. Coach-Created Motivational Climate. In: JOWETT, S. e LAVALLEE, D. (Ed.). Social Psychology of Sport. Champaing: Human Kinetics, p.117-130, 2007.

DUDA, J.; NICHOLLS, J. Dimensions of achievement motivation in schoolwork and sport. Journal of Educational Psychology, Washington, v. 84, p. 290-299, 1992.

\section{DUDA, J.; WHITEHEAD, J. Measurement of} Goal Perspectives in Physical Domain. In: DUDA, J. (Ed.). Advances in Sport and Exercise Psychology Measurement. Morgantown: Fitness Technology Inc, p.21-48, 1998.

DWECK, C. Motivational processes affecting learning. American Psychologist, Washington, v. 41 , p. 1040-1048, 1986.

FONSECA, A.; BRITO, A. A questão da adaptação transcultural de instrumentos para avaliação psicológica em contextos desportivos nacionais - o caso do Task and Ego Orientation in Sport Questionnaire (TEOSQ). Psychologica, Amsterdam, v. 39, p. 95-118, 2005.

GEISINGER, K. Testing and Assessment in Cross-Cultural Psychology. In: GRAHAM, J. e NAGLIERI, J. (Ed.). Handbook of Psychology. Assessment Psychology. New Jersey: John Wiley \& Sons, Inc., p.95-117, 2003.

GEORGIADIS, M.; BIDDLE, S.;

CHATZISARANTIS, N. The mediating role of selfdetermination in the relationship between goal orientation and physical self-worth in greek exercisers. European Journal of Sport Science, London, v. 1, n. 5, p. 1-9, 2001.

GOUDAS, M.; BIDDLE, S. Perceived motivational climate and intrinsic motivation in school physical education classes. European Journal of Psychology of Education, Lisboa, v. 9, p. 241250, 1994.

HAIR, J. et al. Multivariate Data Analysis. 6th. New Jersey: Pearson Educational, Inc., 2006.

HENSON, R.; ROBERTS, J. Use of Exploratory Factor Analysis in Published Research. Common Erros and Some Comment on Improved Practice. Educational and Psychological Measurement, Durham, v. 66, n. 3, p. 393-416, 2006.

HILL, M.; HILL, A. Investigação por

Questionário. Lisboa: Edições Sílabo, 2000. 
HU, L.; BENTLER, P. Cutoff Criteria for Fit Indexes in Covariance Structure Analysis: Conventional Criteria Versus New Alternatives.

Structural Equation Modeling, London, v. 6, n. 1, p. 1-55, 1999.

KAHN, J. Factor Analysis in Counseling Psychology. Research, Trainning, and Practice: Principles, Advances and Applications. The Counseling Psychologist, Thousand Oaks, v. 34, n. 5, p. 684-718, 2006.

KILPATRICK, M.; BARTHOLOMEW, J.; RIEMER, $\mathrm{H}$. The measurement of goal orientations in exercise. Journal of Sport Behavior, Mobile, v. 26, n. 2, p. 121-136, 2003.

KLINE, R. Principles and Practice of Structural Equation Modeling. 2nd. New York: The Guilford Press, 2005.

\section{MAEHR, M.; NICHOLLS, J. Culture and} achievement motivation: A second look. In: WARREN, N. (Ed.). Studies in cross-cultural psychology. New York: Academic Press, v.2, p.221-267, 1980 .

MARDIA, K. Measures of multivariate skewness and kurtosis with applications. Biometrika, London, v. 57, n. 3, p. 519-530, 1970.

MAROCO, J. Análise Estatística com Utilização do SPSS. 3ª . Lisboa: Edições Sílabo, 2007.

MOREIRA, J. Questionários: Teoria e Prática. Coimbra: Livraria Almedina, 2004.

MURCIA, J. et al. Validación preliminar de la escala de percepción del clima motivacional de los iguales (CMI) y la escala de las orientaciones de meta en el ejercicio (GOES) con practicantes españoles de actividades físico-deportivas.

Revista Iberoamericana de Psicología del Ejercicio y el Deporte, Las Palmas de Gran Canaria, v. 1, n. 2, p. 13-28, 2006.

MURCIA, J. et al. Peer's influence on exercise enjoyment: A self-determination theory approach. Journal of Sports Science and Medicine, Bursa, v. 7, n. 23-31, 2008.

NEWTON, M.; DUDA, J.; YIN, Z. Examination of the psychometric properties of the Perceived Motivational Climate in Sport Questionnaire-2 in a sample of female athletes. Journal of Sport Sciences, London, v. 18, p. 275-290, 2000.

NICHOLLS, J. Achievement Motivation: Conceptions of Ability, Subjective Experience, Task Choice, and Performance. Psychological Review, Washington, v. 91, n. 3, p. 328-346, 1984.
NICHOLLS, J. The competitive ethos and democratic education. Cambridge: Harvard University Press, 1989.

NIDEFFER, R.; SAGAL, M. Assessment in Sport Psychology. Morgantown: Fitness Information Technology, 2001.

NTOUMANIS, N.; BIDDLE, S. A review of motivational climate in physical activity. Journal of Sport Sciences, London, v. 17, p. 643-665, 1999.

NTOUMANIS, N.; VAZOU, S. Peer motivational climate in youth sport: Measurement development and validation. Journal of Sport \& Exercise Psychology, Champaign, v. 27, p. 432-455, 2005.

PAPAIOANNOU, A. Development of a questionnaire to measure achivement orientations in physical education. Research Quarterly for Exercise and Sport, Washington, v. 65, n. 1, p. 11-20, 1994.

PAPAIOANNOU, A. Student's perceptions of the physical education class environment for boys and girls and the perceived motivational climate.

Research Quaterly for Exercise and Sport, Washington, v. 69, n. 3, p. 267-275, 1998.

PESTANA, M.; GAGEIRO, J. Análise de Dados para Ciências Sociais. A complementaridade do SPSS. Lisboa: Edições Sílabo, 2005.

PETHERICK, C.; MARKLAND, D. The Development of a Goal Orientation in Exercise Measure (GOEM). Measurement in Physical Education and Exercise Science, London, v. 12, p. 55-71, 2008.

PREACHER, K.; MACCALLUM, R. Repairing Tom Swift's Electric Factor Analysis Machine. Understanding Statistics, London, v. 2, n. 1, p. 13-43, 2003.

ROBERTS, G. Achivement motivation in children's sport. In: NICHOLLS, J. (Ed.). Advances in Motivation and Achievement. The Development of Achievement Motivation. Greenwich, Connecticut: JAI Press Inc, v.3, 1984.

ROBERTS, G. Understanding Motivation. In: ROBERTS, G. (Ed.). Motivation in Sport and Exercise. Champaign, Illinois: Human Kinetics, p.3-29, 1992.

ROBERTS, G. Understanding the dynamics of motivation in physical activity: The influence of achievement goals on motivational processes. In: ROBERTS, G. (Ed.). Advances in Motivation in Sport and Exercise. Champaign-Illinois: Human Kinetics, p.1-50, 2001. 
SATORRA, A.; BENTLER, P. Corrections to test statistics and standard errors in covariance structure analysis. In: EYE, A. e CLOGG, C. (Ed.). Latent variables analysis: Applications for development research. Thousand Oaks: Sage Publications, 1994.

SEIFRIZ, J.; DUDA, J.; CHI, L. The relationship of perceived motivational climate to intrinsic motivation and beliefs about success in basketball. Journal of Sport \& Exercise Psychology, Champaign, v. 14, p. 375-391, 1992.

SMITH, R.; CUMMING, S.; SMOLL, F. Development and Validation of the Motivational Climate Scale for Youth Sports. Journal of Applied Sport Psychology, v. 20, p. 116-136, 2008.

TABACHNICK, B.; FIDELL, L. Using Multivariate Statistics. 2nd. New York: HarperCollins Publishers, 1989.

THOMAS, J.; BARRON, K. A Test of Multiple Achievement Goal Benefits in Physical Education Activities. Journal of Applied Sport Psychology London, v. 18, p. 114-135, 2006.

TREASURE, D.; ROBERTS, G. Perception of Success Questionnaire: Preliminary validation in an adolescent population. Perceptual and Motor Skills, Missoula, v. 79, p. 607-610, 1994.

TREASURE, D.; ROBERTS, G. Applications of achievement goal theory to physical education: Implications for enhancing motivation. Quest, London, v. 47, p. 475-489, 1995.

VALLERAND, R. Vers une méthodologie de validation transculturelle de questionnaires psychologiques: Implications pour la recherche en langue française. Canadian Psychology, Winnipeg, v. 30, n. 4, p.662-680, 1989.

WALLING, M.; DUDA, J.; CHI, L. The Perceived Motivational Climate in Sport Questionnaire: Construct and Predictive Validity. Journal of Sport \& Exercise Psychology, Champaign, v. 15, p. 172-183, 1993.

WHITE, S.; DUDA, J.; HART, S. An exploratory examination of the Parent-Initiated Motivational Climate Questionnaire. Perceptual and Motor Skills, Missoula, v. 75, p. 875-880, 1992.

WORTHINGTON, R.; WHITTAKER, T. Scale Development Research. A Content Analysis and Recommendations for Best Practices. The Counseling Psychologist, Thousand Oaks, v. 34, n. 6, p. 806-838, 2006.

\section{Endereço:}

Luís Cid

Escola Superior de Desporto de Rio Maior Centro de Investigação em Psicologia do Desporto e do Exercício (CIPDE)

Av. Dr. Mário Soares, Pavilhão Multiusos

Rio Maior Portugal

2040-413

e-mail: luiscid@esdrm.ipsantarem.pt

Recebido em: 29 de outubro de 2010.

Aceito em: 25 de outubro de 2012.

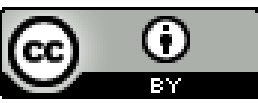

Motriz. Revista de Educação Física. UNESP, Rio Claro, SP, Brasil - elSSN: 1980-6574 - está licenciada sob Creative Commons - Atribuição 3.0 